\title{
On Non-Interactive Simulation of Binary Random Variables
}

\author{
Lei Yu and Vincent Y. F. Tan, Senior Member, IEEE
}

\begin{abstract}
We leverage proof techniques from discrete Fourier analysis and an existing result in coding theory to derive new bounds for the problem of non-interactive simulation of binary random variables. Previous bounds in the literature were derived by applying data processing inequalities concerning maximal correlation or hypercontractivity. We show that our bounds are sharp in some regimes. Indeed, for a specific instance of the problem parameters, our main result resolves an open problem posed by E. Mossel in 2017. As by-products of our analyses, various new properties of the average distance and distance enumerator of binary block codes are established.
\end{abstract}

Index Terms - Non-interactive simulation, distance distribution, average distance, distance enumerator, noise stability, Fourier analysis

\section{INTRODUCTION}

Given a joint distribution $P_{X, Y}$, assume that $(\mathbf{X}, \mathbf{Y}) \sim$ $P_{\mathbf{X}, \mathbf{Y}}:=P_{X, Y}^{n}$ (i.e., $(\mathbf{X}, \mathbf{Y})$ are $n$ i.i.d. copies of $(X, Y) \sim$ $\left.P_{X, Y}\right)$ is a pair of correlated memoryless sources and $(U, V)$ are random variables such that $U-\mathbf{X}-\mathbf{Y}-V$ forms a Markov chain 1 A natural question arises: What are the possible joint distributions of $(U, V)$ ? This problem is termed the noninteractive simulation (NIS) problem [1], [2]. In this paper, we restrict $X, Y, U, V$ to be Boolean random variables (more precisely, random signs) taking values in $\{-1,1\}$ and $P_{X, Y}$ to be a Boolean symmetric distribution (also known as a doubly symmetric binary source), i.e.,

$$
P_{X, Y}=\begin{gathered}
-1 \\
1
\end{gathered}\left[\begin{array}{cc}
\frac{1+\rho}{4} & \frac{1-\rho}{4} \\
\frac{1-\rho}{4} & \frac{1+\rho}{4}
\end{array}\right]
$$

with $\rho \in[-1,1]$. Since the joint distribution of the random variables $U$ and $V$ is entirely determined by the triple of scalars $(\mathbb{P}(U=1), \mathbb{P}(V=1), \mathbb{P}(U=V=1))$, the closure of the set of the possible joint distributions of $(U, V)$ is determined by the following two quantities:

$$
\bar{\Theta}(a, b):=\lim _{n \rightarrow \infty} \bar{\Theta}_{n}(a, b) \quad \text { and } \quad \underline{\Theta}(a, b):=\lim _{n \rightarrow \infty} \underline{\Theta}_{n}(a, b)
$$

This work was supported by a Singapore National Research Foundation (NRF) Fellowship (R-263-000-D02-281) and a Singapore Ministry of Education Tier 2 grant (R-263-000-C83-112).

L. Yu is with the Department of Electrical Engineering and Computer Sciences, University of California, Berkeley, CA, USA, 94720 (e-mail: leiyu.scholar@outlook.com). V. Y. F. Tan is with the Department of Electrical and Computer Engineering and the Department of Mathematics, NUS, Singapore 119076 (e-mail: vtan@nus.edu.sg).

Communicated by A. Gohari, Associate Editor at Large.

Copyright (c) 2021 IEEE. Personal use of this material is permitted. However, permission to use this material for any other purposes must be obtained from the IEEE by sending a request to pubs-permissions@ieee.org.

${ }^{1}$ We say $U, \mathbf{X}, \mathbf{Y}, V$ forms a Markov chain, denoted as $U-\mathbf{X}-\mathbf{Y}-V$, if the joint distribution of $U, \mathbf{X}, \mathbf{Y}, V$ can be written as $P_{U \mid \mathbf{X}} P_{\mathbf{X}, \mathbf{Y}} P_{V \mid \mathbf{Y}}$. where

$$
\bar{\Theta}_{n}(a, b):=\max _{\substack{U, V: U-\mathbf{X}-\mathbf{Y}-V \\ \mathbb{P}(U=1)=a, \mathbb{P}(V=1)=b}} \mathbb{P}(U=V=1)
$$

and $\underline{\Theta}_{n}(a, b)$ is defined similarly but with the maximization replaced by a minimization. The limits here exist, since $\bar{\Theta}_{n}(a, b)$ and $\underline{\Theta}_{n}(a, b)$ are respectively non-increasing and non-decreasing in $n$. Here the fact that $\bar{\Theta}_{n}(a, b)$ and $\underline{\Theta}_{n}(a, b)$ are monotone in $n$ follows from the fact that any pair $(U, V)$ admissible for blocklength $n$ remain admissible for blocklength $n+1$. Furthermore, by replacing $U$ in (2) with $-U$, it follows that $\underline{\Theta}(a, b)=b-\bar{\Theta}(1-a, b)$. Hence the closure of the set of distributions of $(U, V)$ is determined only by $\bar{\Theta}(a, b)$ with $(a, b) \in[0,1]^{2}$ (or by $\underline{\Theta}(a, b)$ and $\bar{\Theta}(a, b)$ with $\left.(a, b) \in\left[0, \frac{1}{2}\right]^{2}\right)$. Although in the definitions of $\underline{\Theta}(a, b)$ and $\bar{\Theta}(a, b), U, V$ are respectively generated by $\mathbf{X}, \mathbf{Y}$ through stochastic maps, in Section III-C we show that stochastic maps can be replaced by deterministic ones (i.e., $U=f(\mathbf{X})$ and $V=g(\mathbf{Y})$ ), without affecting the values of $\underline{\Theta}(a, b)$ and $\bar{\Theta}(a, b)$.

\section{A. Motivations for the NIS Problem}

The study of the NIS problem is motivated by several applications in information theory, cryptography, and stochastic control; see [1]-[3] for example. The quest to determine $\bar{\Theta}(a, b)$ or $\underline{\Theta}(a, b)$ dates back to Witsenhausen's seminal paper [2] published in 1975, in which it was termed the binary decision problem. Witsenhausen's motivation for studying such a problem stemmed from a related information-theoretic problem - the Gács-Körner common information problem [4]. In [2], Witsenhausen applied the tensorization property of the maximal correction (introduced by Hirschfeld [5] and Gebelein [6]) to provide an upper bound for $\bar{\Theta}(a, b)$, which in turn implies a converse result for the Gács-Körner common information. In particular, Witsenhausen addressed the case $a=b=\frac{1}{2}$ by showing that $\bar{\Theta}\left(\frac{1}{2}, \frac{1}{2}\right)$ is attained by a sequence of dictator functions (i.e., $\mathbf{x} \mapsto x_{i}$ for $1 \leq i \leq n$ ). However, the determination of $\bar{\Theta}(a, b)$ (or $\underline{\Theta}(a, b)$ ) has been open for all $(a, b) \in(0,1)^{2} \backslash\left\{\left(\frac{1}{2}, \frac{1}{2}\right)\right\}$. Mossel et al. [7] and O'Donnell [8] as well as Kamath and Anantharam [1] used the forward and reverse hypercontractivity to obtain bounds for the NIS problem; in particular, Kamath and Anantharam's bounds outperform Witsenhausen's maximal correction bounds. In fact, E. Mossel posed the determination of $\bar{\Theta}\left(\frac{1}{4}, \frac{1}{4}\right)$ and $\underline{\Theta}\left(\frac{1}{4}, \frac{1}{4}\right)$ as open problems in 2017 [9]. In this paper, we fully resolve one of these two open problems, namely, the determination of $\bar{\Theta}\left(\frac{1}{4}, \frac{1}{4}\right)$. We also provide new bounds for 
other values of $a$ and $b$. We show that our bounds, which are derived based on discrete Fourier analysis, outperform existing ones [1], [2] in some regimes.

\section{B. Connections to Other Problems}

In the literature, the determination of $\bar{\Theta}_{n}(a, b)$ is also termed the non-interactive correlation distillation (NICD) problem [3], [7], [10]. Deterministic protocols were considered by Mossel, O'Donnell, and other coauthors [7], [10]; while stochastic protocols were permitted and studied in Yang's work [3]. (As mentioned previously, these two scenarios are equivalent.) Moreover, the $k$-terminal version of the NICD problem with $k \geq 3$ was also studied by Mossel and O'Donnell [10]. In this version of the problem, given noise-corrupted versions $\mathbf{X}^{(i)}, 1 \leq i \leq k$ of a source $\mathbf{X}$, it is required to maximize the collision probability $\mathbb{P}\left(f_{1}\left(\mathbf{X}^{(1)}\right)=\ldots=f_{k}\left(\mathbf{X}^{(k)}\right)\right)$ over all balanced Boolean functions $f_{1}, \ldots, f_{k}$. The authors showed that when the number of parties $k$ is 3 , the optimal functions are dictator functions [10]. This extends Witsenhausen's result from the 2-terminal case to the 3-terminal case.

In the study of the NICD problem, it is usually assumed that $a=b$. It is not difficult to show that if $a=b$ are dyadic rationals, then there is a deterministic symmetric protocol $(f, f)$ that attains $\bar{\Theta}_{n}(a, a)$; see Lemma 3 in Section III In other words, if $a$ is a dyadic rational,

$$
\bar{\Theta}_{n}(a, a)=\max _{f: \mathbb{P}(f(\mathbf{X})=1)=a} \mathbb{P}(f(\mathbf{X})=f(\mathbf{Y})=1) .
$$

The determination of this quantity is also called noise stability problem, a problem introduced by Benjamini, Kalai, and Schramm [11]; also see a short survey on [8, p. 68]. Hence the NICD problem (or the maximization part of the NIS problem) can be seen as a generalization of the noise stability problem to the case in which $a$ is not necessarily equal to $b$. Furthermore, the NIS problem is also related to Courtade and Kumar's conjecture on the most informative Boolean function [12] (a weaker version of which was solved by Pichler, Piantanida, and Matz [13]) and the problem studied by Li and Médard [14] concerning the determination of Boolean functions that have the maximum $\alpha$-moment. A variant of NIS problem was also considered by Ordentlich, Polyanskiy and Shayevitz [15]. In [15], the authors considered the scenario in which $a=a_{n}$ and $b=b_{n}$ are allowed to vary with $n$ and both sequences vanish exponentially as $n \rightarrow \infty$. The optimal exponent of this variant for the case $a_{n}=b_{n}$ is implied by an enhanced version of the hypercontractivity inequality provided in [16]. The optimal exponents for arbitrary distributions with finite alphabets and for both symmetric and asymmetric cases have recently been completely characterized by $\mathrm{Yu}$, Anantharam, and Chen [17].

It can also be verified that the NIS problem is equivalent to a coding-theoretic problem concerning the maximizing the distance enumerator between two codes. Moreover, the maximum distance enumerator problem is closely related to the minimum average distance problem proposed in [18]. To describe this connection, we recap a few definitions. A subset $C$ of $\{-1,1\}^{n}$ with size $M$ is called a binary $(n, M)$-code. The average distance of $C$ is defined as the average Hamming distance of every pair of codewords in $C$. Ahlswede and Katona [18] asked a question of determining the minimum of the average distance of $C$ over all sets $C \subseteq\{-1,1\}^{n}$ of a given cardinality $M$. Althöfer and Sillke [19], Fu, Xia, together with other authors [20]-[23], as well as Mounits [24], studied this problem and proved various bounds on the minimum average distance, which are sharp in certain regimes with "large" size of code (e.g., $M=2^{n-1}$ or $2^{n-2}$ ). Ahlswede and Althöfer [25] considered the case in which the size of code increases exponentially in $n$ (with the exponent strictly sandwiched between 0 and 1), and showed that Hamming spheres are asymptotically optimal solutions to the AhlswedeKatona problem as $n \rightarrow \infty$. Mounits [24] studied the case in which the size of the code is "small" (in fact, linear in $n$ ). In this paper, we consider two variants of the AhlswedeKatona's problem. The average distance between two possibly different codes $A, B \subseteq\{-1,1\}^{n}$ is defined as the average Hamming distance between every pair of codewords in $A$ and $B$. The distance enumerator between $A, B \subseteq\{-1,1\}^{n}$ is defined as the generating function of the distance distribution between $A$ and $B$. For $1 \leq M, N \leq 2^{n}$, we are interested in determining the minima and the maxima of the average distance and the distance enumerator between $A$ and $B$ over all sets $A, B \subseteq\{-1,1\}^{n}$ of given cardinalities $M, N$ respectively. The average distance problem considered here extends Ahlswede and Katona's version by allowing the codewords to come from two different codes. On the other hand, by a simple approximation argument (cf. Lemma 1 in Subsection II-C), it can be seen that the distance enumerator problem is asymptotically equivalent to the NIS problem as $n \rightarrow \infty$. The average distance and the distance enumerator are closely related, and hence results on the average distance problem can be applied to the distance enumerator problem and the NIS problem, and vice verse.

\section{Our Main Contributions}

In this paper, we study properties of the average distance and distance enumerator for the case of two binary codes $A$ and $B$. Then by combining the use of discrete Fourier analysis techniques and coding-theoretic results concerning the minimum average distance problem, we derive bounds on the distance enumerator. By leveraging the equivalence between the distance enumerator and the NIS problem, we show that bounds on the former imply some new and nontrivial results on the latter. Moreover, we show that the bounds are sharp for the special case in which $a=b=\frac{1}{4}$. The bounds are tighter than the hypercontractivity bound provided by Kamath and Anantharam [1] in some other regimes, but not in all. For the case in which $a=b=\frac{1}{4}$, our result answers an open problem posed by E. Mossel in 2017 [9].

\section{Paper Organization}

This paper is organized as follows. In Section II] we introduce the definitions of coding-theoretic quantities, including the distance distribution, the average distance, and the distance enumerator. In this section, we also provide the formulation of the NIS problem. In Section III] we study properties of the 
average distance and the distance enumerator. In Section IV these properties are applied to derive bounds on the distance enumerator, or equivalently, bounds for the NIS problem. Finally, in Section $\mathrm{V}$, we provide concluding remarks.

\section{DEFINITIONS AND PRELIMINARIES}

\section{A. Distance Distributions}

For two subsets of the Boolean hypercube (termed codes) $A, B \subseteq\{-1,1\}^{n}$, the distance distribution between $A$ and $B$ is a probability mass function $P^{(A, B)}$ such that for $i \in$ $\{0,1, \ldots, n\}$,

$$
P^{(A, B)}(i):=\frac{1}{|A||B|}\left|\left\{\left(\mathbf{x}, \mathbf{x}^{\prime}\right) \in A \times B: d_{\mathrm{H}}\left(\mathbf{x}, \mathbf{x}^{\prime}\right)=i\right\}\right|,
$$

where $d_{\mathrm{H}}\left(\mathbf{x}, \mathbf{x}^{\prime}\right):=\left|\left\{i: x_{i} \neq x_{i}^{\prime}\right\}\right|$ denotes the Hamming distance between vectors $\mathbf{x}, \mathbf{x}^{\prime}$ (i.e., the number of components of $\mathbf{x}$ and $\mathbf{x}^{\prime}$ that differ). It is clear that $P^{(A, B)}(0)=$ $\frac{|A \cap B|}{|A||B|}, \sum_{i=0}^{n} P^{(A, B)}(i)=1$, and $P^{(A, B)}(i) \geq 0$ for $i \in$ $\{0,1, \ldots, n\}$.

Define the $k$-th distance moment and the distance enumerator between $A, B \subseteq\{-1,1\}^{n}$ respectively as

$$
\begin{aligned}
D_{k}(A, B) & :=\frac{1}{|A||B|} \sum_{\mathbf{x} \in A} \sum_{\mathbf{x}^{\prime} \in B} d_{\mathrm{H}}^{k}\left(\mathbf{x}, \mathbf{x}^{\prime}\right) \\
& =\sum_{i=0}^{n} P^{(A, B)}(i) \cdot i^{k},
\end{aligned}
$$

and

$$
\begin{aligned}
\Gamma_{z}(A, B) & :=\frac{1}{|A||B|} \sum_{\mathbf{x} \in A} \sum_{\mathbf{x}^{\prime} \in B} z^{d_{\mathrm{H}}\left(\mathbf{x}, \mathbf{x}^{\prime}\right)} \\
& =\sum_{i=0}^{n} P^{(A, B)}(i) \cdot z^{i}, \quad z \geq 0 .
\end{aligned}
$$

Clearly, $D_{k}(A, B)$ and $\Gamma_{z}(A, B)$ are respectively the $k$-th moment and the generating function of $P^{(A, B)}$, and hence they are closely related. For $k=1, D(A, B):=D_{1}(A, B)$ corresponds to the average distance.

The dual distance distribution between codes $A, B$ is defined by

$$
\begin{aligned}
Q^{(A, B)}(i):= & \frac{1}{|A||B|} \sum_{\mathbf{u} \in\{0,1\}^{n}: w_{\mathrm{H}}(\mathbf{u})=i}\left(\sum_{\mathbf{x} \in \frac{A+1}{2}}(-1)^{\langle\mathbf{u}, \mathbf{x}\rangle}\right) \\
& \times\left(\sum_{\mathbf{x} \in \frac{B+1}{2}}(-1)^{\langle\mathbf{u}, \mathbf{x}\rangle}\right), i=0,1, \ldots, n,
\end{aligned}
$$

where $w_{\mathrm{H}}(\mathbf{u}):=d_{\mathrm{H}}(\mathbf{u}, \mathbf{0})$ denotes the Hamming weight (i.e., the number of nonzero components) of a vector $\mathbf{u}$, and $\langle\mathbf{u}, \mathbf{x}\rangle:=\left(\sum_{i=1}^{n} u_{i} x_{i}\right) \bmod 2$ denotes the inner product of vectors $\mathbf{u}, \mathbf{x} \in \mathbb{F}_{2}^{n}$. Clearly, $Q^{(A, B)}(0)=1$. Note that $Q^{(A, B)}(i)$ can be negative for some $i$, and $\sum_{i=0}^{n} Q^{(A, B)}(i)$ can be smaller or larger than 1 . Hence in general, $Q^{(A, B)}$ is not a probability mass function.

The dual distance enumerator between $A, B$ is defined as

$$
\Pi_{z}(A, B):=\sum_{i=0}^{n} Q^{(A, B)}(i) \cdot z^{i}, \quad z \geq 0 .
$$

It is easy to verify that the following MacWilliams-Delsarte identities hold.

$$
\begin{aligned}
& \Pi_{z}(A, B)=(1+z)^{n} \Gamma_{\frac{1-z}{1+z}}(A, B) \\
& \Gamma_{z}(A, B)=\left(\frac{1+z}{2}\right)^{n} \Pi_{\frac{1-z}{1+z}}(A, B) .
\end{aligned}
$$

If $A=B$, then $Q^{(A, A)}(i) \geq 0$, and by (5), $\sum_{i=0}^{n} Q^{(A, A)}(i)=$ $\frac{2^{n}}{|A|}$. Hence for this case, $\frac{|A|}{2^{n}} Q^{(A, A)}(\cdot)$ is a probability mass function. Furthermore, the MacWilliams-Delsarte identities for the case $A=B$ can be found in [20], [26].

For brevity, we utilize the following abbreviations: $P^{(A)}:=$ $P^{(A, A)}, Q^{(A)}:=Q^{(A, A)}, D_{k}(A):=D_{k}(A, A), \Gamma_{z}(A):=$ $\Gamma_{z}(A, A)$ and $\Pi_{z}(A):=\Pi_{z}(A, A)$.

\section{B. Basics of Discrete Fourier Analysis}

We now provide a brief primer on the discrete Fourier analysis needed in this paper. We also discuss its relationship to the dual distance distribution. Define $[n]:=\{1,2, \ldots, n\}$. Given the basis $\chi_{S}(\mathbf{x}):=\prod_{i \in S} x_{i}$ for $S \subseteq[n]$, for a Boolean function $f:\{-1,1\}^{n} \rightarrow\{-1,1\}$, define its Fourier coefficients as

$$
\hat{f}_{S}:=\mathbb{E}_{\mathbf{X} \sim \operatorname{Unif}\{-1,1\}^{n}}\left[f(\mathbf{X}) \chi_{S}(\mathbf{X})\right], \quad S \subseteq[n] .
$$

Then the Fourier expansion of a Boolean function $f$ (cf. [8 Eqn. (1.6)]) is

$$
f(\mathbf{x})=\sum_{S \subseteq[n]} \hat{f}_{S} \chi_{S}(\mathbf{x})
$$

By definition, it is easy to verify that for two codes $A, B \subseteq$ $\{-1,1\}^{n}$ with their $\{-1,1\}$-valued indicator functions denoted as $f=2 \cdot \mathbf{1}_{A}-1, g=2 \cdot \mathbf{1}_{B}-1$, the dual distance distribution $Q^{(A, B)}$ and the Fourier coefficients $\hat{f}_{S}, \hat{g}_{S}$ satisfy the following relationship ${ }^{2}$

$$
Q^{(A, B)}(k)=\left\{\begin{array}{ll}
1, & k=0 \\
\frac{1}{4 a b} \sum_{S:|S|=k} \hat{f}_{S} \hat{g}_{S}, & 1 \leq k \leq n
\end{array} .\right.
$$

\section{Non-Interactive Simulation}

Let $(X, Y)$ be a pair of binary random variables on $\{-1,1\}$ with the distribution given in (1), where $\rho \in[-1,1]$ denotes the correlation between $X$ and $Y$. Assume $(\mathbf{X}, \mathbf{Y})$ are $n$ i.i.d. copies of $(X, Y)$. Then we focus on the following NIS problem: Given $a, b \in[0,1]$, what is the possible range of the collision probability $\mathbb{P}(U=V)$ for all Boolean random variables $U, V$ (or equivalently, conditional Boolean distributions $\left.P_{U \mid \mathbf{X}}, P_{V \mid \mathbf{Y}}\right)$ such that $U-\mathbf{X}-\mathbf{Y}-V$ and $\mathbb{P}(U=1)=a, \mathbb{P}(V=1)=b$ ? Obviously, $\mathbb{P}(U=V)=$ $1+2 \mathbb{P}(U=V=1)-a-b$. Hence it suffices to consider the possible range (i.e., the maximum and minimum) of $\mathbb{P}(U=V=1)$. Furthermore, the maximum and minimum

${ }^{2}$ Note that if $f=\mathbf{1}_{A}, g=\mathbf{1}_{B}$ are $\{0,1\}$-valued indicator functions, then (8) reduces to the simpler relation:

$$
Q^{(A, B)}(k)=\frac{1}{a b} \sum_{S:|S|=k} \hat{f}_{S} \hat{g}_{S}, \quad 0 \leq k \leq n
$$


$\mathbb{P}(U=V=1)$ can be approximated by replacing the conditional Boolean distributions $P_{U \mid \mathbf{X}}, P_{V \mid \mathbf{Y}}$ with Boolean functions, as shown by the following lemma. For dyadic rationals $a_{n}:=\frac{\left\lfloor 2^{n} a\right\rfloor}{2^{n}}$ and $b_{n}:=\frac{\left\lfloor 2^{n} b\right\rfloor}{2^{n}}$ with $\lfloor x\rfloor$ denoting the maximum integer not larger than $x$, define

$$
\Delta_{n}^{+}:=\bar{\Theta}_{n}(a, b)-\max _{\substack{f, g: \mathbb{P}(f(\mathbf{X})=1)=a_{n}, \mathbb{P}(g(\mathbf{Y})=1)=b_{n}}} \mathbb{P}(f(\mathbf{X})=g(\mathbf{Y})=1),
$$

with $\bar{\Theta}_{n}(a, b)$ defined in (2), and define $\Delta_{n}^{-}$as the RHS of (9) with both maximizations (including the one in the definition of $\left.\bar{\Theta}_{n}(a, b)\right)$ replaced by minimizations.

Lemma 1. It holds that

$$
0 \leq \Delta_{n}^{+}, \Delta_{n}^{-} \leq a-a_{n}+b-b_{n} \leq 2^{-(n-1)}
$$

In particular, if $n, a, b$ are such that $a=\frac{M}{2^{n}}$ and $b=\frac{N}{2^{n}}$ with some $M, N \in \mathbb{N}$, then $\Delta_{n}^{+}=\Delta_{n}^{-}=0$, i.e., the maximum or minimum $\mathbb{P}(U=V=1)$ is attained by some Boolean functions $f, g:\{-1,1\}^{n} \rightarrow\{-1,1\}$ such that $U=f(\mathbf{X})$ and $V=g(\mathbf{Y})$.

Proof: First, we claim that for arbitrary $n \in \mathbb{N}$ and $a, b \in[0,1]$, the maximum or minimum $\mathbb{P}(U=V=1)$ over all Boolean random variables $U, V$ such that $U-\mathbf{X}-\mathbf{Y}-V$ and $\mathbb{P}(U=1)=a, \mathbb{P}(V=1)=b$ is attained by some pair of conditional Boolean distributions $\left(P_{U \mid \mathbf{X}}, P_{V \mid \mathbf{Y}}\right)$ with the following properties: There exists at most one $\mathbf{x}_{0} \in\{-1,1\}^{n}$ such that $0<P_{U \mid \mathbf{X}}\left(1 \mid \mathbf{x}_{0}\right)<1$ and there exists at most one $\mathbf{y}_{0} \in\{-1,1\}^{n}$ such that $0<P_{V \mid \mathbf{Y}}\left(1 \mid \mathbf{y}_{0}\right)<1$. This claim follows from the following argument. First, observe that $\mathbb{P}(U=V=1)$ can be written as

$$
\mathbb{P}(U=V=1)=\sum_{\mathbf{x} \in\{-1,1\}^{n}} \lambda_{\mathbf{x}} P_{U \mid \mathbf{X}}(1 \mid \mathbf{x})
$$

where

$$
\lambda_{\mathbf{x}}=\sum_{\mathbf{y}} P_{\mathbf{X}, \mathbf{Y}}(\mathbf{x}, \mathbf{y}) P_{V \mid \mathbf{Y}}(1 \mid \mathbf{y}) .
$$

Hence, for fixed $P_{V \mid \mathbf{Y}}$, the maximization or minimization of $\mathbb{P}(U=V=1)$ can be considered as a linear programming problem with the decision variable being the vector $\left(P_{U \mid \mathbf{X}}(1 \mid \mathbf{x}): \mathbf{x} \in\{-1,1\}^{n}\right)$ whose components satisfy $0 \leq P_{U \mid \mathbf{X}}(1 \mid \mathbf{x}) \leq 1$ and $\mathbb{P}(U=1)=$ $\frac{1}{2^{n}} \sum_{\mathbf{x} \in\{-1,1\}^{n}} P_{U \mid \mathbf{X}}(1 \mid \mathbf{x})=a$. These constraints correspond to the intersection of the hyperplane $\left\{\mathbf{t} \in \mathbb{R}^{2^{n}}\right.$ : $\left.\frac{1}{2^{n}} \sum_{i=1}^{2^{n}} t_{i}=a\right\}$ and the hypercube $[0,1]^{2^{n}}$. This intersection is a convex polygon. Hence, by the fundamental theorem of linear programming [27], the maximum and minimum of $\mathbb{P}(U=V=1)$ are attained at the corners (i.e., extreme points) of this polygon. Observe that each corner point of the polygon lies between two adjacent corner points of the cube. Hence they are precisely the points $P_{U \mid \mathbf{X}}(1 \mid \cdot)$ such that $0 \leq P_{U \mid \mathbf{X}}\left(1 \mid \mathbf{x}_{0}\right) \leq 1$ for some $\mathbf{x}_{0}$ and $P_{U \mid \mathbf{X}}(1 \mid \mathbf{x})=0$ or 1 for all $\mathbf{x} \neq \mathbf{x}_{0}$.

Furthermore, for arbitrary $n \in \mathbb{N}$ and $a, b \in[0,1]$, observe that the contribution of $P_{U \mid \mathbf{X}}\left(1 \mid \mathbf{x}_{0}\right)$ to the probability $\mathbb{P}(U=1)$ is $P_{U \mid \mathbf{X}}\left(1 \mid \mathbf{x}_{0}\right) P_{\mathbf{X}}\left(\mathbf{x}_{0}\right)=a-a_{n}$. The contribution of $P_{U \mid \mathbf{X}}\left(1 \mid \mathbf{x}_{0}\right)$ to the probability $\mathbb{P}(U=V=1)$ is
$P_{U \mid \mathbf{X}}\left(1 \mid \mathbf{x}_{0}\right) P_{\mathbf{X}}\left(\mathbf{x}_{0}\right) P_{V \mid \mathbf{X}}\left(1 \mid \mathbf{x}_{0}\right)$ which is lower bounded by 0 and upper bounded by $P_{U \mid \mathbf{X}}\left(1 \mid \mathbf{x}_{0}\right) P_{\mathbf{X}}\left(\mathbf{x}_{0}\right)=a-a_{n}$. Choose $f(\mathbf{x})=2 P_{U \mid \mathbf{X}}(1 \mid \mathbf{x})-1$ for $\mathbf{x} \neq \mathbf{x}_{0}$ and $f\left(\mathbf{x}_{0}\right)=-1$ for $\mathbf{x}_{0}$ such that $0<P_{U \mid X}\left(1 \mid \mathbf{x}_{0}\right)<1$ provided such an $\mathbf{x}_{0}$ exists; otherwise, choose $f(\mathbf{x})=2 P_{U \mid \mathbf{X}}(1 \mid \mathbf{x})-1$ for all $\mathbf{x}$. Apply the same argument above to $P_{V \mid \mathbf{Y}}$, and choose $g$ in a similar way. This concludes the proof of Lemma 1 .

From this lemma, we have $\lim _{n \rightarrow \infty} \Delta_{n}^{+}=\lim _{n \rightarrow \infty} \Delta_{n}^{-}=$ 0 . Hence in this paper, we restrict the maximization (resp. minimization) of $\mathbb{P}(U=V=1)$ over Markov chains $U-\mathbf{X}-$ $\mathbf{Y}-V$ to the maximization (resp. minimization) over pairs of Boolean functions $f, g:\{-1,1\}^{n} \rightarrow\{-1,1\}$ such that $U=f(\mathbf{X})$ and $V=g(\mathbf{Y})$. That is, we consider the following key question:

Given $a=\frac{M}{2^{n}}$ and $b=\frac{N}{2^{n}}$ for some $M, N \in \mathbb{N}$, what are the maximum and minimum values of the probability $\mathbb{P}(f(\mathbf{X})=g(\mathbf{Y})=1)$ over all Boolean functions $f, g:\{-1,1\}^{n} \rightarrow\{-1,1\}$ such that $\mathbb{P}(f(\mathbf{X})=1)=a$ and $\mathbb{P}(g(\mathbf{Y})=1)=b$ ?

Denote $A:=\{\mathbf{x}: f(\mathbf{x})=1\}$ and $B:=\{\mathbf{x}: g(\mathbf{x})=1\}$. Then for $a=\frac{M}{2^{n}}$ and $b=\frac{N}{2^{n}}$ with some $M, N \in \mathbb{N}$, $\mathbb{P}(f(\mathbf{X})=1)=a$ and $\mathbb{P}(g(\mathbf{Y})=1)=b$ imply that $|A|=M$ and $|B|=N$. By (5), we have

$$
\begin{aligned}
& \mathbb{P}(f(\mathbf{X})=g(\mathbf{Y})=1) \\
& =\sum_{\mathbf{x} \in A} \sum_{\mathbf{x}^{\prime} \in B}\left(\frac{1-\rho}{4}\right)^{d_{\mathrm{H}}\left(\mathbf{x}, \mathbf{x}^{\prime}\right)}\left(\frac{1+\rho}{4}\right)^{n-d_{\mathrm{H}}\left(\mathbf{x}, \mathbf{x}^{\prime}\right)} \\
& =\left(\frac{1+\rho}{4}\right)^{n} \sum_{\mathbf{x} \in A} \sum_{\mathbf{x}^{\prime} \in B}\left(\frac{1-\rho}{1+\rho}\right)^{d_{\mathrm{H}}\left(\mathbf{x}, \mathbf{x}^{\prime}\right)} \\
& =a b(1+\rho)^{n} \Gamma_{\frac{1-\rho}{1+\rho}}(A, B) \\
& =a b \Pi_{\rho}(A, B)
\end{aligned}
$$

Hence, solving the Boolean function version of the NIS problem is equivalent to maximizing and minimizing $\Gamma_{\frac{1-\rho}{1+\rho}}(A, B)$ or $\Pi_{\rho}(A, B)$ over all subsets $A, B$ such that $|A| \stackrel{1+\rho}{=} M$ and $|B|=N$.

\section{BASic Properties of Distance Moments AND DISTANCE ENUMERATORS}

For a set $A \subseteq\{-1,1\}^{n}$, define its complement and componentwise complement respectively as $A^{c}:=\{-1,1\}^{n} \backslash A$, and $A^{\star}:=\{-\mathbf{x}: \mathbf{x} \in A\}$, where $-\mathbf{x}=\left(-x_{1},-x_{2}, \ldots,-x_{n}\right)$ is the componentwise negation of $\mathbf{x}$. Some salient properties of the average distances and distance enumerators are summarized as follows:

Lemma 2. For $A, B \subseteq\{-1,1\}^{n}$, the following hold.

$$
\begin{aligned}
&|A| D(A, B)+\left|A^{c}\right| D\left(A^{c}, B\right)=n 2^{n-1} ; \\
& D_{k}\left(A^{\star}, B\right)=\sum_{i=0}^{k}\left(\begin{array}{c}
k \\
i
\end{array}\right) n^{k-i}(-1)^{i} D_{i}(A, B) ; \\
&|A| \Gamma_{z}(A, B)+\left|A^{c}\right| \Gamma_{z}\left(A^{c}, B\right)=(1+z)^{n} ; \\
& \Gamma_{z}\left(A^{\star}, B\right)=z^{n} \Gamma_{\frac{1}{z}}(A, B) .
\end{aligned}
$$

Lemma 2 is straightforward, hence the proof is omitted. 
Remark 1. For $k=1$, (14) implies that

$$
D(A, B)+D\left(A^{\star}, B\right)=n .
$$

Lemma 3. For $A, B \subseteq\{-1,1\}^{n}$, the following hold.

$$
\begin{aligned}
\left|\frac{n}{2}-D(A, B)\right| & \leq \sqrt{\left(\frac{n}{2}-D(A)\right)\left(\frac{n}{2}-D(B)\right)} \\
& \leq \frac{n}{2}-\frac{1}{2}(D(A)+D(B)) .
\end{aligned}
$$

For $0 \leq z \leq 1$,

$$
\Gamma_{z}(A, B) \leq \sqrt{\Gamma_{z}(A) \Gamma_{z}(B)} \leq \frac{1}{2}\left(\Gamma_{z}(A)+\Gamma_{z}(B)\right)
$$

and for $z \geq 1$,

$$
\begin{aligned}
\Gamma_{z}(A, B) & \leq \sqrt{\Gamma_{z}\left(A^{\star}, A\right) \Gamma_{z}\left(B^{\star}, B\right)} \\
& \leq \frac{1}{2}\left(\Gamma_{z}\left(A^{\star}, A\right)+\Gamma_{z}\left(B^{\star}, B\right)\right) .
\end{aligned}
$$

Proof: Denote $f:=2 \cdot \mathbf{1}_{A}-1$ and $g:=2 \cdot \mathbf{1}_{B}-1$. Both $\frac{n}{2}-D(A, B)$ and $\Gamma_{z}(A, B)$ can be written as bilinear functions of $\hat{f}_{S}$ and $\hat{g}_{S}$. Then applying the Cauchy-Schwarz inequality, the claims in the lemma follow. In the following, we provide the detailed proof.

Define

$$
\begin{aligned}
& a:=|A| / 2^{n}=\mathbb{P}(f(\mathbf{X})=1)=\frac{1+\hat{f}_{\emptyset}}{2}, \\
& b:=|B| / 2^{n}=\mathbb{P}(g(\mathbf{X})=1)=\frac{1+\hat{g}_{\emptyset}}{2}, \\
& \theta_{\rho}:=\frac{1}{4} \sum_{S \subseteq[n]:|S| \geq 1} \hat{f}_{S} \hat{g}_{S} \rho^{|S|} .
\end{aligned}
$$

In analogy to [8. Plancherel's Theorem and Proposition 1.9], the inner product between $f(\mathbf{X})$ and $g(\mathbf{Y})$ satisfies

$$
\mathbb{E}[f(\mathbf{X}) g(\mathbf{Y})]=\hat{f}_{\emptyset} \hat{g}_{\emptyset}+4 \theta_{\rho}=2 \mathbb{P}(f(\mathbf{X})=g(\mathbf{Y}))-1 .
$$

Defining $\bar{t}:=1-t$ for $t \in[0,1]$ and following by (22) and the fact that

$$
\mathbb{P}(f(\mathbf{X})=g(\mathbf{Y}))=1+2 \mathbb{P}(f(\mathbf{X})=g(\mathbf{Y})=1)-a-b,
$$

we can write

$$
\mathbb{P}(f(\mathbf{X})=g(\mathbf{Y})=1)=a b+\theta_{\rho},
$$

which further implies

$$
\begin{aligned}
& \mathbb{P}(f(\mathbf{X})=-g(\mathbf{Y})=1)=a \bar{b}-\theta_{\rho} \\
& \mathbb{P}(-f(\mathbf{X})=g(\mathbf{Y})=1)=\bar{a} b-\theta_{\rho} \\
& \mathbb{P}(f(\mathbf{X})=g(\mathbf{Y})=-1)=\bar{a} \bar{b}+\theta_{\rho} .
\end{aligned}
$$

Combining (23), (12), and (8) yields the following identity:

$$
\begin{aligned}
a b(1+\rho)^{n} \Gamma_{\frac{1-\rho}{1+\rho}}(A, B) & =a b \Pi_{\rho}(A, B) \\
& =a b+\frac{1}{4} \sum_{S:|S| \geq 1} \hat{f}_{S} \hat{g}_{S} \rho^{|S|} .
\end{aligned}
$$

By the Cauchy-Schwarz inequality, we have for any $0 \leq k \leq$ $n$,

$$
\left|\sum_{S:|S|=k} \hat{f}_{S} \hat{g}_{S}\right| \leq \sqrt{\left(\sum_{S:|S|=k} \hat{f}_{S}^{2}\right)\left(\sum_{S:|S|=k} \hat{g}_{S}^{2}\right)} .
$$

Now consider the case of $k=1$. For any $i \in[n]$,

$$
\hat{f}_{\{i\}}=\frac{1}{2^{n}}\left(\sum_{\mathbf{x} \in A} x_{i}-\sum_{\mathbf{x} \in A^{c}} x_{i}\right)=\frac{2}{2^{n}} \sum_{\mathbf{x} \in A} x_{i},
$$

since $\sum_{\mathbf{x} \in A} x_{i}+\sum_{\mathbf{x} \in A^{c}} x_{i}=0$. Similarly, for any $i \in[n]$,

$$
\hat{g}_{\{i\}}=\frac{2}{2^{n}} \sum_{\mathbf{x} \in B} x_{i} .
$$

Therefore,

$$
\begin{aligned}
c:=\sum_{S:|S|=1} \hat{f}_{S} \hat{g}_{S} & =\sum_{i=1}^{n} \frac{1}{4^{n-1}} \sum_{\mathbf{x} \in A} x_{i} \sum_{\mathbf{x}^{\prime} \in B} x_{i}^{\prime} \\
& =\frac{1}{4^{n-1}} \sum_{\mathbf{x} \in A} \sum_{\mathbf{x}^{\prime} \in B}\left(n-2 d_{\mathrm{H}}\left(\mathbf{x}, \mathbf{x}^{\prime}\right)\right) \\
& =4 a b(n-2 D(A, B))
\end{aligned}
$$

where $a=\frac{|A|}{2^{n}}$ and $b=\frac{|B|}{2^{n}}$. Combining (28) for $k=1$ with (32) (and its symmetric versions with $B$ replaced by $A$ or $A$ replaced by $B$ ) yields

$$
\begin{aligned}
\left|\frac{n}{2}-D(A, B)\right| & \leq \sqrt{\left(\frac{n}{2}-D(A)\right)\left(\frac{n}{2}-D(B)\right)} \\
& \leq \frac{n}{2}-\frac{1}{2}(D(A)+D(B))
\end{aligned}
$$

Now we prove inequality 19 . By the Cauchy-Schwarz inequality, we obtain that for $\rho \in[0,1]$,

$$
\begin{aligned}
& a b+\frac{1}{4} \sum_{S:|S| \geq 1} \hat{f}_{S} \hat{g}_{S} \rho^{|S|} \\
& \leq \sqrt{\left(a^{2}+\frac{1}{4} \sum_{S:|S| \geq 1} \hat{f}_{S}^{2} \rho^{|S|}\right)\left(b^{2}+\frac{1}{4} \sum_{S:|S| \geq 1} \hat{g}_{S}^{2} \rho^{|S|}\right) .} .
\end{aligned}
$$

Combining 27) with 33 yields

$$
\begin{aligned}
\Gamma_{\frac{1-\rho}{1+\rho}}(A, B) & \leq \sqrt{\Gamma_{\frac{1-\rho}{1+\rho}}(A) \Gamma_{\frac{1-\rho}{1+\rho}}(B)} \\
& \leq \frac{1}{2}\left(\Gamma_{\frac{1-\rho}{1+\rho}}(A)+\Gamma_{\frac{1-\rho}{1+\rho}}(B)\right) .
\end{aligned}
$$

Setting $z:=\frac{1-\rho}{1+\rho} \in[0,1]$, we obtain that for $0 \leq z \leq 1$,

$$
\Gamma_{z}(A, B) \leq \sqrt{\Gamma_{z}(A) \Gamma_{z}(B)} \leq \frac{1}{2}\left(\Gamma_{z}(A)+\Gamma_{z}(B)\right) .
$$

Inequality (20) follows by combining (19) with (16).

In fact, in the proof of Lemma 3, we prove the following inequality: The dual distance distribution between any codes $A$ and $B$ satisfies

$$
\left|Q^{(A, B)}(k)\right| \leq \sqrt{Q^{(A)}(k) Q^{(B)}(k)}, \quad 0 \leq k \leq n .
$$

Inequality (18) corresponds to inequality (34) with $k=1$.

Lemma 4. For $1 \leq M \leq 2^{n}$,

$$
\begin{aligned}
\min _{A, B:|A|=|B|=M} D(A, B) & =\min _{A:|A|=M} D(A), \\
\max _{A, B:|A|=|B|=M} D(A, B) & =n-\min _{A:|A|=M} D(A) .
\end{aligned}
$$


For $0 \leq z \leq 1$

$$
\max _{A, B:|A|=|B|=M} \Gamma_{z}(A, B)=\max _{A:|A|=M} \Gamma_{z}(A) ;
$$

and for $z \geq 1$,

$$
\max _{A, B:|A|=|B|=M} \Gamma_{z}(A, B)=\max _{A:|A|=M} \Gamma_{z}\left(A^{\star}, A\right) .
$$

Proof: By (18),

$$
D(A, B) \geq \frac{1}{2}(D(A)+D(B)) .
$$

Minimizing both sides over $A, B$ such that $|A|=|B|=M$, we obtain

$$
\min _{A, B:|A|=|B|=M} D(A, B) \geq \min _{A:|A|=M} D(A) .
$$

On the other hand, obviously,

$$
\min _{A, B:|A|=|B|=M} D(A, B) \leq \min _{A:|A|=M} D(A) .
$$

Hence, (35) follows.

Equation (36) follow since by (17) and 35,

$$
\begin{aligned}
\max _{A, B:|A|=|B|=M} D(A, B) & =n-\min _{A, B:|A|=|B|=M} D\left(A^{\star}, B\right) \\
& =n-\min _{A:|A|=M} D(A),
\end{aligned}
$$

Equations (37) and (38) respectively follow from (19) and 20.

Fu, Wei, and Yeung [23, Thm. 4] considered the average distance between codewords in the same set (i.e., restricting $A$ and $B$ to be identical), showed that for $a:=\frac{M}{2^{n}} \leq \frac{1}{2}$,

$$
\min _{A:|A|=M} D(A) \geq \frac{n}{2}-\frac{1}{4 a},
$$

by using a linear programming approach. Here equality in (40) holds for $M=2^{n-1}$ or $2^{n-2}$ by setting $A$ to be a subcube (e.g., $A=\{1\} \times\{-1,1\}^{n-1}$ for $M=2^{n-1}$ and $A=\{1\}^{2} \times\{-1,1\}^{n-2}$ for $M=2^{n-2}$ ). Combining this result with Lemmas 2 and 3 yields the following bounds on the average distance between two possibly non-identical sets.

Lemma 5. For $1 \leq M, N \leq 2^{n}$, we have

$$
\begin{array}{r}
\min _{A, B:|A|=M,|B|=N} D(A, B) \geq \frac{n}{2}-\frac{\sqrt{(a \wedge \bar{a})(b \wedge \bar{b})}}{4 a b}, \\
\max _{A, B:|A|=M,|B|=N} D(A, B) \leq \frac{n}{2}+\frac{\sqrt{(a \wedge \bar{a})(b \wedge \bar{b})}}{4 a b},
\end{array}
$$

where $a:=\frac{M}{2^{n}}, b:=\frac{N}{2^{n}}$, and $x \wedge y:=\min \{x, y\}$. Equalities in (41) and (42) hold for $M \in\left\{2^{n-1}, 2^{n-2}, 3 \cdot 2^{n-2}\right\}$ and $N=M$ or $2^{n}-M$, by setting $A$ to a subcube, and $B=A$ or $\left(A^{c}\right)^{\star}$ for 41 ); $B=A$ or $A^{c}$ for $(42$ ).

Proof: By (17), Inequalities (41) and (42) are in fact equivalent. Hence, it suffices to prove 41.

Inequality (41) for the case $a, b \leq \frac{1}{2}$ follows since by (18) and 40,

$\left|\frac{n}{2}-D(A, B)\right| \leq \sqrt{\left(\frac{n}{2}-D(A)\right)\left(\frac{n}{2}-D(B)\right)} \leq \frac{1}{4 \sqrt{a b}}$.
We now consider the case $b \leq \frac{1}{2}<a$. By (13) and (17),

$$
\begin{aligned}
& |A| D(A, B)+\left|A^{c}\right|\left(n-D\left(\left(A^{c}\right)^{\star}, B\right)\right) \\
& \quad=|A| D(A, B)+\left|A^{c}\right| D\left(A^{c}, B\right)=n 2^{n-1},
\end{aligned}
$$

which, combined with (41) for the case $a, b \leq \frac{1}{2}$, implies the case $b \leq \frac{1}{2}<a$. By symmetry, the case $b \leq \frac{1}{2}<a$ also holds.

We next consider the case $a, b>\frac{1}{2}$. By (13) we have

$$
\begin{aligned}
|A| D(A, B)+\left|A^{c}\right| D\left(A^{c}, B\right) & =n 2^{n-1} \\
|B| D\left(B, A^{c}\right)+\left|B^{c}\right| D\left(B^{c}, A^{c}\right) & =n 2^{n-1} .
\end{aligned}
$$

Since $D\left(B, A^{c}\right)=D\left(A^{c}, B\right)$, we obtain

$|A||B| D(A, B)-\left|A^{c}\right|\left|B^{c}\right| D\left(A^{c}, B^{c}\right)=\left(|B|-\left|A^{c}\right|\right) n 2^{n-1}$.

Combining (43) with inequality 41 for the case $a, b \leq \frac{1}{2}$ yields the case $a, b>\frac{1}{2}$.

\section{Non-Interactive Simulation}

\section{A. Non-Interactive Simulation}

In this section, we derive bounds on $\Gamma_{z}(A, B)$ and $\Pi_{z}(A, B)$ for $z \geq 0$ and for $A, B$ such that $|A|=M$ and $|B|=N$. By (12), this is equivalent to bounding $\mathbb{P}(f(\mathbf{X})=g(\mathbf{Y})=1)$ for Boolean functions $f, g$ such that $\mathbb{P}(f(\mathbf{X})=1)=a$ and $\mathbb{P}(g(\mathbf{Y})=1)=b$ where $a=\frac{M}{2^{n}}$ and $b=\frac{N}{2^{n}}$. Without loss of generality, we may assume $0 \leq a \leq b \leq \frac{1}{2}$ (or $0 \leq M \leq N \leq 2^{n-1}$ ) and $\rho \in[0,1]$. This is because that if $\rho<0$, then we can then replace $-X$ by $X$; if $a>\frac{1}{2}$ or $b>\frac{1}{2}$, replace $-f$ by $f$ or $-g$ by $g$. For Boolean functions $f, g$ such that $\mathbb{P}(f(\mathbf{X})=1)=a$ and $\mathbb{P}(g(\mathbf{Y})=1)=b$, define

$$
q:=\mathbb{P}(f(\mathbf{X})=g(\mathbf{Y})=1)
$$

Then $q$ can be bounded as follows.

Theorem 1 (Bounds on $q$ ).

$$
\max \left\{\Upsilon_{1}^{\mathrm{LB}}, \Upsilon_{2}^{\mathrm{LB}}\right\} \leq q \leq \min \left\{\Upsilon_{1}^{\mathrm{UB}}, \Upsilon_{2}^{\mathrm{UB}}\right\}
$$

where

$$
\begin{aligned}
& \Upsilon_{1}^{\mathrm{LB}}:=\max \left\{0, a b-\frac{\sqrt{a b}}{2} \rho-\frac{a b+\sqrt{a \bar{a} b \bar{b}}}{2} \rho^{2}\right\} \\
& \Upsilon_{2}^{\mathrm{LB}}:=\max \left\{0, a b-\frac{\sqrt{a b}}{2} \rho-\frac{a+b-2 a b-\sqrt{a b}}{2} \rho^{2}\right\} \\
& \Upsilon_{1}^{\mathrm{UB}}:=\min \left\{a, a b+\frac{\sqrt{a b}}{2} \rho+\frac{a \bar{b}+\sqrt{a \bar{a} b \bar{b}}-\sqrt{a b}}{2} \rho^{2}\right\} \\
& \Upsilon_{2}^{\mathrm{UB}}:=\sqrt{\theta^{+}(a) \theta^{+}(b)}
\end{aligned}
$$

with

$$
\theta^{+}(t):=t^{2}+\frac{t}{2} \rho+\left(\frac{t}{2}-t^{2}\right) \rho^{2}
$$

Remark 2. For $a=b, \Upsilon_{1}^{\mathrm{LB}} \leq \Upsilon_{2}^{\mathrm{LB}}$, and for fixed $b$ and sufficiently small $a, \Upsilon_{1}^{\mathrm{LB}} \geq \Upsilon_{2}^{\mathrm{LB}}$. Hence $\Upsilon_{1}^{\mathrm{LB}} \leq \Upsilon_{2}^{\mathrm{LB}}$ or $\Upsilon_{1}^{\mathrm{LB}} \geq \Upsilon_{2}^{\mathrm{LB}}$ does not always hold. Similarly, $\Upsilon_{1}^{\mathrm{UB}} \leq \Upsilon_{2}^{\mathrm{UB}}$ or $\Upsilon_{1}^{\mathrm{UB}} \geq \Upsilon_{2}^{\mathrm{UB}}$ also does not always hold. 
Proof: The main idea in the proof is as follows. Recall from 23 that

$$
q=a b+\theta_{\rho} \quad \text { with } \quad \theta_{\rho}=\frac{1}{4} \sum_{k=1}^{n}\left(\sum_{S:|S|=k} \hat{f}_{S} \hat{g}_{S}\right) \rho^{k},
$$

which implies that bounding $q$ is equivalent to bounding $\theta_{\rho}$. By Parseval's theorem and the Cauchy-Schwarz inequality, the sum of absolute values of coefficients, $\sum_{k=1}^{n}\left|\sum_{S:|S|=k} \hat{f}_{S} \hat{g}_{S}\right|$, is bounded. On the other hand, recall that (see (32) for $k=1, \sum_{S:|S|=1} \hat{f}_{S} \hat{g}_{S}=$ $4 a b(n-2 D(A, B))$. Hence by Lemma 5 , we can bound the term $\sum_{S:|S|=1} \hat{f}_{S} \hat{g}_{S}$, which in turns implies bounds for $q$. Furthermore, in order to further improve bounds for $q$ in certain cases, we incorporate the "partition" technique from Pichler, Piantanida, and Matz [13] in our proof.

We now provide the details of the proof. By the nonnegativity of the probabilities given in $23-(26)$,

$$
-a b \leq \theta_{\rho} \leq a \bar{b}
$$

As in the proof of Lemma 3, we write $A:=\{\mathbf{x}: f(\mathbf{x})=1\}$ and $B:=\{\mathbf{x}: g(\mathbf{x})=1\}$. Then by Lemma 5, we obtain that

$$
\left|\frac{n}{2}-D(A, B)\right| \leq \frac{1}{4 \sqrt{a b}}
$$

since $a, b \leq \frac{1}{2}$ as assumed. Hence $c$, as defined in (31), satisfies

$$
|c| \leq 2 \sqrt{a b} .
$$

As in the proof of Lemma 3. we denote the Fourier coefficients of $f$ and $g$ as $\hat{f}_{S}$ and $\hat{g}_{S}$, respectively; see (7). To bound $\theta_{\rho}$, we partition the set $\{S \subseteq[n]:|S| \geq 2\}$ into $\mathcal{P}:=\left\{S \subseteq[n]:|S| \geq 2, \hat{f}_{S} \hat{g}_{S} \geq 0\right\}$ and $\mathcal{N}:=\{S \subseteq$ $\left.[n]:|S| \geq 2, \hat{f}_{S} \hat{g}_{S}<0\right\}$. This idea for doing this partitioning comes from [13]. We define

$$
\tau^{+}:=\frac{1}{4} \sum_{S \in \mathcal{P}} \hat{f}_{S} \hat{g}_{S}, \quad \text { and } \quad \tau^{-}:=\frac{1}{4} \sum_{S \in \mathcal{N}} \hat{f}_{S} \hat{g}_{S} .
$$

Then for $\rho=1, \theta_{1}$ can be written as

$$
\theta_{1}=\frac{1}{4} c+\tau^{+}+\tau^{-}
$$

and by definition,

$$
\frac{1}{4} c \rho+\rho^{2} \tau^{-} \leq \theta_{\rho} \leq \frac{1}{4} c \rho+\rho^{2} \tau^{+} .
$$

Hence to bound $\theta_{\rho}$, it suffices to upper bound $\tau^{+}$and lower bound $\tau^{-}$, which will be done by respectively bounding $\tau^{+}-$ $\tau^{-}$and $\tau^{+}+\tau^{-}$. We next do this.

We first apply the Cauchy-Schwarz inequality to bound $\tau^{+}-\tau^{-}$.

$$
\begin{aligned}
\frac{1}{4} c+\tau^{+}-\tau^{-} & \leq \frac{1}{4} \sum_{S:|S| \geq 1}\left|\hat{f}_{S}\right|\left|\hat{g}_{S}\right| \\
& \leq \frac{1}{4} \sqrt{\left(\sum_{S:|S| \geq 1} \hat{f}_{S}^{2}\right)\left(\sum_{S:|S| \geq 1} \hat{g}_{S}^{2}\right)} \\
& =\frac{1}{4} \sqrt{\left(1-\hat{f}_{\emptyset}^{2}\right)\left(1-\hat{g}_{\emptyset}^{2}\right)} \\
& =\sqrt{a \bar{a} b \bar{b}}
\end{aligned}
$$

where (51) follows from Parseval's theorem [8]. Hence

$$
\tau^{+}-\tau^{-} \leq \sqrt{a \bar{a} b \bar{b}}-\frac{1}{4} c .
$$

We next bound $\tau^{+}+\tau^{-}$. Since $-a b \leq \theta_{1} \leq a \bar{b}$ (see (46)), by substituting (49) in to these inequalities, we have

$$
-a b-\frac{1}{4} c \leq \tau^{+}+\tau^{-} \leq a \bar{b}-\frac{1}{4} c .
$$

Combining (52) and (53), we can bound $\tau^{+}$and $\tau^{-}$as follows:

$$
\tau^{+} \leq \frac{a \bar{b}+\sqrt{a \bar{a} b \bar{b}}}{2}-\frac{1}{4} c, \quad \tau^{-} \geq-\frac{a b+\sqrt{a \bar{a} b \bar{b}}}{2} .
$$

Combining the bounds in (54) with (50) yields that

$$
\theta_{\rho} \in\left[\widehat{\theta}_{\rho}^{-}, \widehat{\theta}_{\rho}^{+}\right] \subseteq\left[\theta_{\rho}^{-}, \theta_{\rho}^{+}\right],
$$

where, from (48),

$$
\begin{aligned}
\widehat{\theta}_{\rho}^{-} & :=\max \left\{-a b, \frac{1}{4} c \rho-\frac{a b+\sqrt{a \bar{a} b \bar{b}}}{2} \rho^{2}\right\} \\
& \geq \max \left\{-a b,-\frac{\sqrt{a b}}{2} \rho-\frac{a b+\sqrt{a \bar{a} b \bar{b}}}{2} \rho^{2}\right\}=: \theta_{\rho}^{-}, \\
\widehat{\theta}_{\rho}^{+} & :=\min \left\{a \bar{b}, \frac{1}{4} c \rho+\left(\frac{a \bar{b}+\sqrt{a \bar{a} b \bar{b}}}{2}-\frac{1}{4} c\right) \rho^{2}\right\} \\
& \leq \min \left\{a \bar{b}, \frac{\sqrt{a b}}{2} \rho+\frac{a \bar{b}+\sqrt{a \bar{a} b \bar{b}}-\sqrt{a b}}{2} \rho^{2}\right\}=: \theta_{\rho}^{+} .
\end{aligned}
$$

Combining (55) with (45) gives us the lower bound $\Upsilon_{1}^{L B}$ and upper bound $\Upsilon_{1}^{\mathrm{UB}}$.

We next prove that $q$ is lower bounded by $\Upsilon_{2}^{L B}$. Starting from the definition of $\theta_{\rho}$ in 21, we obtain that

$$
\begin{aligned}
\theta_{\rho} & \geq \frac{1}{4} c \rho-\frac{1}{4} \sum_{i=2}^{n}\left|\sum_{S:|S|=i} \hat{f}_{S} \hat{g}_{S}\right||\rho|^{i} \\
& \geq-\frac{1}{4}|c| \rho-\frac{1}{4}\left(\sum_{i=2}^{n}\left|\sum_{S:|S|=i} \hat{f}_{S} \hat{g}_{S}\right|\right) \rho^{2} .
\end{aligned}
$$

We bound the term $\sum_{i=2}^{n}\left|\sum_{S:|S|=i} \hat{f}_{S} \hat{g}_{S}\right|$ by observing that

$$
\begin{aligned}
& (2 a-1)(2 b-1)+|c|+\sum_{i=2}^{n}\left|\sum_{S:|S|=i} \hat{f}_{S} \hat{g}_{S}\right| \\
& =\sum_{i=0}^{n}\left|\sum_{S:|S|=i} \hat{f}_{S} \hat{g}_{S}\right| \\
& \leq \sum_{S \subseteq[n]}\left|\hat{f}_{S}\right|\left|\hat{g}_{S}\right| \\
& \leq \sqrt{\left(\sum_{S \subseteq[n]} \hat{f}_{S}^{2}\right)\left(\sum_{S \subseteq[n]} \hat{g}_{S}^{2}\right)}=1 .
\end{aligned}
$$

Hence

$$
\begin{aligned}
\sum_{i=2}^{n}\left|\sum_{S:|S|=i} \hat{f}_{S} \hat{g}_{S}\right| & \leq 1-(2 a-1)(2 b-1)-|c| \\
& =2 a+2 b-4 a b-|c| .
\end{aligned}
$$


Substituting (57) into [56), we obtain

$$
\begin{aligned}
\theta_{\rho} & \geq-\frac{1}{4}|c| \rho-\frac{1}{4}(2 a+2 b-4 a b-|c|) \rho^{2} \\
& \geq-\frac{\sqrt{a b}}{2} \rho-\frac{a+b-2 a b-\sqrt{a b}}{2} \rho^{2},
\end{aligned}
$$

where (58) follows since $|c| \leq 2 \sqrt{a b}$ (see (48)). On the other hand, $\theta_{\rho} \geq-a b$. Hence,

$$
\theta_{\rho} \geq \max \left\{-a b,-\frac{\sqrt{a b}}{2} \rho-\frac{a+b-2 a b-\sqrt{a b}}{2} \rho^{2}\right\} .
$$

Substituting it into (45), we obtain the lower bound $\Upsilon_{2}^{\mathrm{LB}}$.

We finally prove the upper bound $\Upsilon_{2}^{\mathrm{UB}}$. Denote

$$
\begin{aligned}
& q_{f}:=\mathbb{P}(f(\mathbf{X})=f(\mathbf{Y})=1), \\
& q_{g}:=\mathbb{P}(g(\mathbf{X})=g(\mathbf{Y})=1) .
\end{aligned}
$$

From (11), we express $q, q_{f}, q_{g}$ in terms of the distance distributions as follows:

$$
\begin{aligned}
q & =a b(1+\rho)^{n} \Gamma_{\frac{1-\rho}{1+\rho}}(A, B) \\
q_{f} & =a^{2}(1+\rho)^{n} \Gamma_{\frac{1-\rho}{1+\rho}}(A) \\
q_{g} & =b^{2}(1+\rho)^{n} \Gamma_{\frac{1-\rho}{1+\rho}}(B) .
\end{aligned}
$$

Combining these expressions with (19) yields that

$$
q \leq \sqrt{q_{f} q_{g}} .
$$

Applying the upper bound $\Upsilon_{1}^{\mathrm{UB}}$ respectively to $q_{f}$ and $q_{g}$, we obtain

$$
q_{f} \leq \theta^{+}(a), \quad q_{g} \leq \theta^{+}(b) .
$$

Combining (59) and 60) yields the desired bound $\Upsilon_{2}^{\mathrm{UB}}$.

Corollary 1. If $a=b$, then

$$
\theta^{-}(a) \leq q \leq \theta^{+}(a)
$$

where

$$
\theta^{-}(t):=\max \left\{0, t^{2}-\frac{t}{2} \rho-\left(\frac{t}{2}-t^{2}\right) \rho^{2}\right\},
$$

and $\theta^{+}(t)$ is defined in (44). In particular, for $a=b=\frac{1}{2}$,

$$
\frac{1-\rho}{4} \leq q \leq \frac{1+\rho}{4}
$$

and for $a=b=\frac{1}{4}$,

$$
\frac{1-2 \rho-\rho^{2}}{16} \leq q \leq\left(\frac{1+\rho}{4}\right)^{2} .
$$

The bounds in (61] are not new; see [2]. However, the bounds in 62 are new. Note that the bounds in (61) are sharp since the upper bound is attained by the functions $f(\mathbf{x})=g(\mathbf{x})$ and $f(\mathbf{x})=1$ if $x_{1}=1$ and -1 otherwise, and the lower bound is attained by the functions $f(\mathbf{x})=g(-\mathbf{x})$ and $f(\mathbf{x})=1$ if $x_{1}=1$ and -1 otherwise.

For $a=b=\frac{1}{4}$, the upper bound in (62) is sharp and attained by the functions $f(\mathbf{x})=g(\mathbf{x})$ and $f(\mathbf{x})=1$ if $x_{1}=$ $x_{2}=1$ and -1 otherwise. This answers one of two open problems posed by Mossel [9]. These problems concern the determination of the maximum $q$ and minimum $q$ when $a=$ $b=\frac{1}{4}$; we resolve the maximum part of the question. For the minimum $q$, we do not believe that our lower bound in (62) is tight. In fact, for $a=b=\frac{1}{4}$, we conjecture that $q$ is, in fact, lower bounded by $\left(\frac{1-\rho}{4}\right)^{2}$. If this conjecture is true, then this lower bound is sharp since it is attained by the functions $f(\mathbf{x})=g(-\mathbf{x})$ and $f(\mathbf{x})=1$ if $x_{1}=x_{2}=1$ and -1 otherwise. Furthermore, one may conjecture that for $a=b=2^{-i}, i \in \mathbb{N},\left(\frac{1-\rho}{4}\right)^{i} \leq q \leq\left(\frac{1+\rho}{4}\right)^{i}$. However, this does not hold, since when $a=b$ are small, the $q$ induced by functions $f=g=2 \cdot \mathbf{1}_{B}-1$ with $B \subseteq\{-1,1\}^{n}$ denoting a Hamming ball is strictly larger than $\left(\frac{1+\rho}{4}\right)^{i}$, and moreover, the induced $q$ by such a pair $(f, g)$ is close to the optimal value [8, Remark 10.2]. A similar conclusion also holds for the case of minimizing $q$.

\section{B. Comparisons to Other Bounds}

Define the maximal correlation between two random variables $X, Y$ as

$$
\rho_{\mathrm{m}}(X ; Y):=\sup _{f, g} \mathbb{E}[f(X) g(Y)],
$$

where the supremum is taken over all real-valued Borelmeasurable functions $f$ and $g$ such that $\mathbb{E}[f(X)]=$ $\mathbb{E}[g(Y)]=0$ and $\mathbb{E}\left[f^{2}(X)\right]=\mathbb{E}\left[g^{2}(Y)\right]=1$. When specialized to the binary case,

$\rho_{\mathrm{m}}(X ; Y)=|\rho(X ; Y)|=\frac{\left|P_{X, Y}(1,1)-P_{X}(1) P_{Y}(1)\right|}{\sqrt{P_{X}(1) P_{X}(-1) P_{Y}(1) P_{Y}(-1)}}$.

This quantity was first introduced by Hirschfeld [5] and Gebelein [6], then studied by Rényi [28], and it has been exploited to provide a necessary condition for the NIS problem [1], [2]. Non-interactive simulation of $(U, V) \sim P_{U V}$ using $(\mathbf{X}, \mathbf{Y}) \sim P_{X, Y}^{n}$ is possible only if $\rho_{\mathrm{m}}(U ; V) \leq \rho_{\mathrm{m}}(X ; Y)$. When specialized to the binary case, it leads to the following bounds on $q$.

Proposition 1 (Maximal Correlation Bounds [2]). One has

$$
a b-\sqrt{a \bar{a} b \bar{b}} \rho \leq q \leq a b+\sqrt{a \bar{a} b \bar{b}} \rho .
$$

By comparing the maximal correlation upper bound above to $\Upsilon_{1}^{\mathrm{UB}}$, we get $\Upsilon_{1}^{\mathrm{UB}} \leq a b+\sqrt{a \bar{a} b \bar{b}} \rho$. Equality here holds only when $a=b=\frac{1}{2}$. Hence our upper bound $\Upsilon_{1}^{\mathrm{UB}}$ is tighter than the maximal correlation upper bound. On the other hand, comparing the maximal correlation lower bound above to $\Upsilon_{2}^{\mathrm{LB}}$, we get that for $a=b$ (i.e., the symmetric case), $\Upsilon_{2}^{\mathrm{LB}} \geq a b-\sqrt{a \bar{a} b \bar{b}} \rho$, i.e., our bound $\Upsilon_{2}^{\mathrm{LB}}$ is tighter than the maximal correlation lower bound; for the case $a \leq b=\frac{1}{2}$, $\Upsilon_{2}^{\mathrm{LB}} \leq a b-\sqrt{a \bar{a} b \bar{b}} \rho$, i.e., our bound $\Upsilon_{2}^{\mathrm{LB}}$ is looser than the maximal correlation lower bound. Hence, in general, our lower bound $\Upsilon_{2}^{\mathrm{LB}}$ is neither tighter nor looser than the maximal correlation lower bound.

Hypercontractivity (which yields the forward and reverse hypercontractivity inequalities) is a powerful tool in studying extremal problems, especially in high-dimensional spaces. Recently, this tool has seen increasing use for investigating various problems in information theory; see [1], [8], [29][32] for example. In [1], [7], [8], Mossel, O’Donnell, Kamath, 
et al. applied it to derive bounds for the NIS problem. For example, the following bounds are provided in [1]. More precisely, the following upper bound was derived by using the forward hypercontractivity inequality, and the following lower bound by the reverse hypercontractivity inequality. These are Eqns. (28) and (29) in [1].

Proposition 2 (Hypercontractivity Bounds [1]). One has

$$
\begin{aligned}
& \sup _{s, t>0,(s-1)(t-1)(\kappa-1)<0} \varphi_{a, b}(s, t, \kappa) \\
& \leq q \leq \inf _{s, t>0,(s-1)(t-1)(\kappa-1)>0} \varphi_{a, b}(s, t, \kappa),
\end{aligned}
$$

where

$\varphi_{a, b}(s, t, \kappa):=\frac{\left(s^{\kappa^{\prime}} a+\bar{a}\right)^{\frac{1}{\kappa^{\prime}}}\left(t^{\kappa} b+\bar{b}\right)^{\frac{1}{\kappa}}-1}{(s-1)(t-1)}-\frac{a}{t-1}-\frac{b}{s-1}$

with $\kappa^{\prime}:=1+\frac{\rho^{2}}{\kappa-1}$.

Our bounds in Corollary 1 , the maximal correlation bounds in Proposition 1, and the hypercontractivity bounds in Proposition 2 are plotted in Fig. 1 for the symmetric case in which $a=b$. We set $\rho=0.1,0.5$, and 0.9 . These numerical results show that the hypercontractivity lower bound is neither tighter nor looser than our lower bound in general, and the hypercontractivity upper bound is also neither tighter nor looser than our upper bound in general. More specifically, the hypercontractivity bounds are tighter than ours for small $a$, while our bounds are tighter than the hypercontractivity ones for large $a$ (and they coincide for $a=1 / 2$ ). These figures also verify that both our bounds and the hypercontractivity bounds are uniformly tighter than the maximal correlation bounds for the symmetric case. The fact that for the symmetric case, the hypercontractivity bounds are uniformly tighter than the maximal correlation bounds has been rigorously proven in [1. Corollary 1].

\section{CONCLUding REMARKS}

In the proof of Theorem 1, we applied Fu-Wei-Yeung's bound [23] on the average distance (given in (40) to bound the distance enumerator. On the other hand, as shown in Fig. 1. the hypercontractivity bounds on the distance enumerator are tighter than our bounds in Theorem 1 in some regimes. Hence, intuitively, the hypercontractivity bounds can in turn be applied to obtain nontrivial bounds on the average distance. In fact, the following bound derived by Chang [8], [33] is derived by leveraging such an idea: For $1 \leq M \leq 2^{n}$ and $a:=\frac{M}{2^{n}}$,

$$
\min _{A:|A|=M} D(A) \geq \frac{n}{2}-\log \frac{1}{a} .
$$

Chang's bound was proven by the "single-function" version of the hypercontractivity inequality. Replacing the "singlefunction" version of the hypercontractivity inequality with the "two-function" version (i.e., the inequalities in Proposition 2), one can obtain the following tighter bound (since $\psi(a) \leq$ $\left.\log \frac{1}{a}\right)$ : For $1 \leq M \leq 2^{n}$, we have

$$
\min _{A:|A|=M} D(A) \geq \frac{n}{2}-\psi(a)
$$
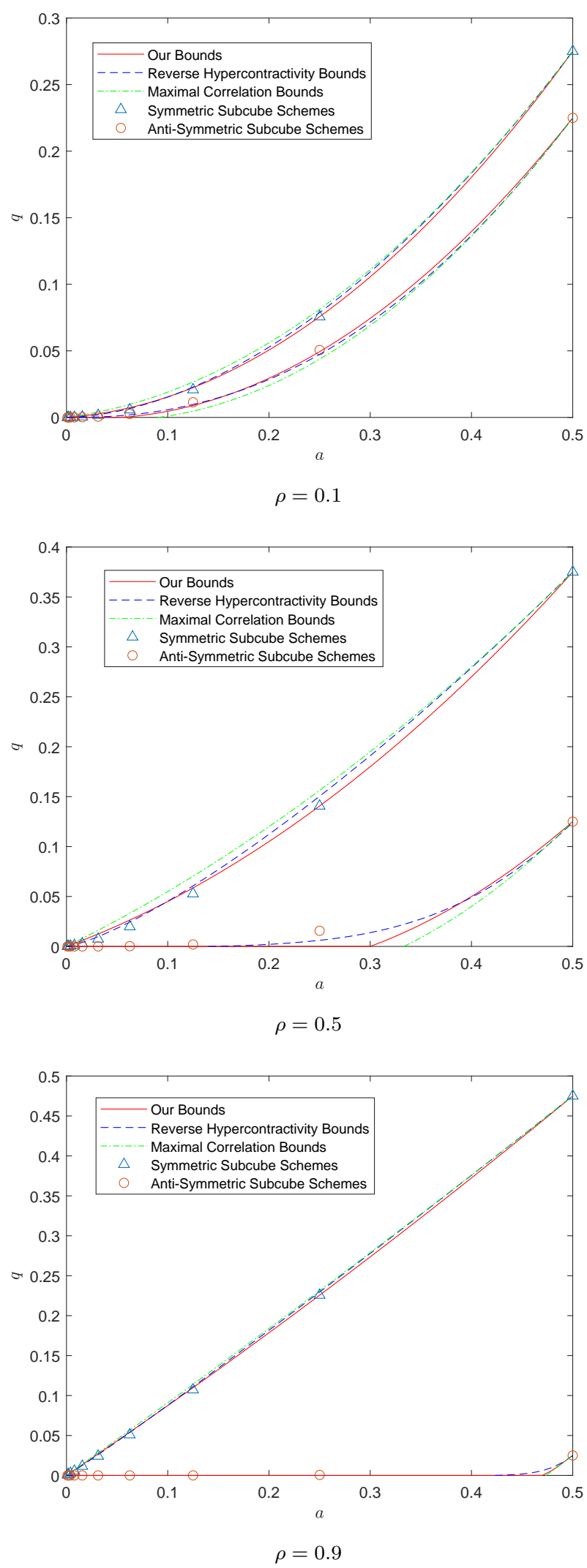

Fig. 1. Illustration of our bounds in Corollary 1 the maximal correlation bounds in Proposition 1 and the hypercontractivity bounds in Proposition 2 The upper set of curves correspond to upper bounds, while the lower set of curves correspond to lower bounds. The symmetric subcube schemes correspond to the maps $\left\{\left(f_{i}, g_{i}\right)\right\}_{i=1}^{\infty}$ such that $f_{i}(\mathbf{x})=g_{i}(\mathbf{x})$ and $f_{i}(\mathbf{x})=1$ if $x_{1}=x_{2}=\ldots=x_{i}=1$ and -1 otherwise. The anti-symmetric subcube schemes correspond to the maps $\left\{\left(f_{i}, g_{i}\right)\right\}_{i=1}^{\infty}$ such that $f_{i}(\mathbf{x})=g_{i}(-\mathbf{x})$ and $f_{i}(\mathbf{x})=1$ if $x_{1}=x_{2}=\ldots=x_{i}=1$ and -1 otherwise. 
where

$$
\psi(a):=\inf _{t>0, t \neq 1} \frac{(t a+\bar{a})[a t \log t-(t a+\bar{a}) \log (t a+\bar{a})]}{a^{2}(t-1)^{2}} .
$$

In Fu-Wei-Yeung's linear programming bound given in (40), $\psi(a)$ is replaced with $\frac{1}{4 a}$. Furthermore, in [34], the present authors provided an improved linear programming bound on the average distance. This bound is better than Fu-Wei-Yeung's bound when $a<\frac{1}{4}$ and is the best over all existing bounds for $a=\frac{1}{8}$. This improved linear programming bound was also used to derive an improved bound for the NIS problem in [34].

In addition, one may wonder whether the hypercontractivity bound for the NIS problem in Proposition 2 can be improved by using data processing inequalities (DPIs) arising from the family of $\Phi$-strong data processing inequality (SDPI) constants [35, Theorem 19] or $\Phi$-ribbons [35, Theorem 12] which were proposed by Beigi and Gohari [35]. We term bounds resulting from these two DPIs as the $\Phi$-SDPI bound and the $\Phi$-ribbon bound respectively. Then, Kamath-Anantharam's hypercontractivity upper bound in Proposition 2 is a special case of the $\Phi$-ribbon bound with $\Phi(t)=t \log t[35]$. We conjecture that in our setting (i.e., DSBS $(X, Y)$ and binary $U$ and $V$ ), under the common tensorization condition that $\Phi \in \mathscr{F}$ with the family of functions $\mathscr{F}$ defined in [35, Definition 6], the hypercontractivity upper bound in Proposition 2 is the tightest over all $\Phi$-ribbon bounds. In fact, the first author of this paper and V. Anantharam have shown a weaker version of this conjecture. That is, the bound derived from the DPI $s^{*}(U, V) \leq s^{*}(X, Y)$ with $s^{*}(W, Z)$ denoting the hypercontractivity constant of $(W, Z)$, is the tightest $\Phi$-SDPI bound over all $\Phi \in \mathscr{F}[36]$. This will be the content of a future paper [36].

\section{Acknowledgements}

The authors thank the associate editor Prof. Amin Gohari and the two reviewers for insightful comments that have helped to improve the paper.

\section{REFERENCES}

[1] S. Kamath and V. Anantharam. On non-interactive simulation of joint distributions. IEEE Trans. Inf. Theory, 62(6):3419-3435, 2016.

[2] H. S. Witsenhausen. On sequences of pairs of dependent random variables. SIAM Journal on Applied Mathematics, 28(1):100-113, 1975.

[3] K. Yang. On the (im)possibility of non-interactive correlation distillation. Theoretical Computer Science, 382(2):157-166, 2007.

[4] P. Gács and J. Körner. Common information is far less than mutual information. Problems of Control and Information Theory, 2(2):149$162,1973$.

[5] H. O. Hirschfeld. A connection between correlation and contingency. In Mathematical Proceedings of the Cambridge Philosophical Society, volume 31, pages 520-524. Cambridge University Press, 1935.

[6] H. Gebelein. Das statistische problem der korrelation als variations-und eigenwertproblem und sein zusammenhang mit der ausgleichsrechnung. ZAMM-Journal of Applied Mathematics and Mechanics/Zeitschrift für Angewandte Mathematik und Mechanik, 21(6):364-379, 1941.

[7] E. Mossel, R. O’Donnell, O. Regev, J. E. Steif, and B. Sudakov. Noninteractive correlation distillation, inhomogeneous Markov chains, and the reverse Bonami-Beckner inequality. Israel Journal of Mathematics, 154(1):299-336, 2006.

[8] R. O'Donnell. Analysis of Boolean Functions. Cambridge University Press, 2014.
[9] E. Mossel. Mixing in product spaces. http://math.mit.edu/ elmos/slides.pdf, 2017.

[10] E. Mossel and R. O'Donnell. Coin flipping from a cosmic source: On error correction of truly random bits. Random Structures \& Algorithms, 26(4):418-436, 2005.

[11] I. Benjamini, G. Kalai, and O. Schramm. Noise sensitivity of Boolean functions and applications to percolation. Publications Mathématiques de l'Institut des Hautes Etudes Scientifiques, 90(1):5-43, 1999.

[12] T. A. Courtade and G. R. Kumar. Which Boolean functions maximize mutual information on noisy inputs? IEEE Trans. Inf. Theory, 60(8):4515-4525, 2014

[13] G. Pichler, P. Piantanida, and G. Matz. Dictator functions maximize mutual information. The Annals of Applied Probability, 28(5):30943101, 2018.

[14] J. Li and M. Médard. Boolean functions: Noise stability, non-interactive correlation distillation, and mutual information. IEEE Trans. Inf. Theory, 67(2):778-789, 2021.

[15] O. Ordentlich, Y. Polyanskiy, and O. Shayevitz. A note on the probability of rectangles for correlated binary strings. IEEE Trans. Inf. Theory, 66(12):7878-7886, 2020

[16] N. Kirshner and A. Samorodnitsky. A moment ratio bound for polynomials and some extremal properties of Krawchouk polynomials and Hamming spheres. arXiv preprint arXiv:1909.11929, 2019.

[17] L. Yu, V. Anantharam, and J. Chen. Graphs of joint types, noninteractive simulation, and stronger hypercontractivity. under preparation, 2021.

[18] R. Ahlswede and G. O. H. Katona. Contributions to the geometry of Hamming spaces. Discrete Mathematics, 17(1):1-22, 1977.

[19] I. Althöfer and T. Sillke. An "average distance" inequality for large subsets of the cube. Journal of Combinatorial Theory, Series B, 56(2):296-301, 1992.

[20] S. Xia and F.-W. Fu. On the average Hamming distance for binary codes. Discrete Applied Mathematics, 89(1-3):269-276, 1998.

[21] F.-W. Fu and S.-Y. Shen. On the expectation and variance of Hamming distance between two iid random vectors. Acta Mathematicae Applicatae Sinica, 13(3):243-250, 1997.

[22] F.-W. Fu, T. Klove, and S.-Y. Shen. On the Hamming distance between two iid random n-tuples over a finite set. IEEE Trans. Inf. Theory, 45(2):803-807, 1999.

[23] F.-W. Fu, V. K. Wei, and R. W. Yeung. On the minimum average distance of binary codes: Linear programming approach. Discrete Applied Mathematics, 111(3):263-281, 2001.

[24] B. Mounits. Lower bounds on the minimum average distance of binary codes. Discrete Mathematics, 308(24):6241-6253, 2008.

[25] R. Ahlswede and I. Althöfer. The asymptotic behavior of diameters in the average. Journal of Combinatorial Theory, Series B, 61(2):167-177, 1994.

[26] F. J. MacWilliams and N. J. A. Sloane. The Theory of Error-Correcting Codes, volume 16. North Holland, Elsevier, 1977.

[27] D. P. Bertsimas and J. N. Tsitsiklis. Introduction to Linear Optimization. Athena Scientific, 1997.

[28] A. Rényi. On measures of dependence. Acta Mathematica Hungarica, 10(3-4):441-451, 1959

[29] V. Anantharam, A. Gohari, S. Kamath, and C. Nair. On hypercontractivity and a data processing inequality. In 2014 IEEE International Symposium on Information Theory, pages 3022-3026. IEEE, 2014.

[30] S. Beigi and A. Gohari. Monotone measures for non-local correlations. IEEE Trans. Inf. Theory, 61(9):5185-5208, 2015.

[31] J. Liu, R. van Handel, and S. Verdú. Second-order converses via reverse hypercontractivity. Mathematical Statistics and Learning, 2(2):103-163, 2019.

[32] M. Raginsky. Strong data processing inequalities and $\Phi$-Sobolev inequalities for discrete channels. IEEE Trans. Inf. Theory, 62(6):3355$3389,2016$.

[33] M.-C. Chang. A polynomial bound in Freiman's theorem. Duke Mathematical Journal, 113(3):399-419, 2002.

[34] L. Yu and V. Y. F. Tan. An improved linear programming bound on the average distance of a binary code. arXiv preprint arXiv:1910.09416, 2019.

[35] S. Beigi and A. Gohari. $\Phi$-entropic measures of correlation. IEEE Trans. Inf. Theory, 64(4):2193-2211, 2018.

[36] L. Yu and V. Anantharam. The hypercontractivity constant is the largest tensorized SDPI constant for binary sources. under preparation, 2021. 
Lei Yu received the B.E. and Ph.D. degrees, both in electronic engineering, from University of Science and Technology of China (USTC) in 2010 and 2015, respectively. From 2015 to 2017, he was a postdoctoral researcher at the Department of Electronic Engineering and Information Science (EEIS), USTC. From 2017 to 2019, he was a research fellow at the Department of Electrical and Computer Engineering, National University of Singapore. Currently, he is a postdoc at the Department of Electrical Engineering and Computer Sciences, University of California, Berkeley. His research interests lie in the intersection of information theory, probability theory, and combinatorics.

Vincent Y. F. Tan (S'07-M'11-SM'15) was born in Singapore in 1981. He is currently a Dean's Chair Associate Professor in the Department of Electrical and Computer Engineering and the Department of Mathematics at the National University of Singapore (NUS). He received the B.A. and M.Eng. degrees in Electrical and Information Sciences from Cambridge University in 2005 and the Ph.D. degree in Electrical Engineering and Computer Science (EECS) from the Massachusetts Institute of Technology (MIT) in 2011. His research interests include information theory, machine learning, and statistical signal processing.

Dr. Tan received the MIT EECS Jin-Au Kong outstanding doctoral thesis prize in 2011, the NUS Young Investigator Award in 2014, the Singapore National Research Foundation (NRF) Fellowship (Class of 2018) and the NUS Young Researcher Award in 2019. He was also an IEEE Information Theory Society Distinguished Lecturer for 2018/9. He is currently serving as an Associate Editor of the IEEE Transactions on Signal Processing and an Associate Editor of Machine Learning for the IEEE Transactions on Information Theory. He is a member of the IEEE Information Theory Society Board of Governors. 